\title{
ImageCLEF 2020: Multimedia Retrieval in Lifelogging, Medical, Nature, and Internet Applications
}

\author{
Bogdan Ionescu ${ }^{1(\bowtie)}$, Henning Müller ${ }^{2}$, Renaud Péteri ${ }^{3}$, \\ Duc-Tien Dang-Nguyen ${ }^{4}$, Liting Zhou ${ }^{5}$, Luca Piras ${ }^{6}$, Michael Riegler ${ }^{7}$, \\ Pål Halvorsen ${ }^{7}$, Minh-Triet Tran ${ }^{8}$, Mathias Lux ${ }^{9}$, Cathal Gurrin ${ }^{5}$, \\ Jon Chamberlain ${ }^{10}$, Adrian Clark ${ }^{10}$, Antonio Campello ${ }^{11}$, \\ Alba G. Seco de Herrera ${ }^{10}$, Asma Ben Abacha ${ }^{12}$, Vivek Datla ${ }^{13}$, \\ Sadid A. Hasan ${ }^{14}$, Joey Liu ${ }^{13}$, Dina Demner-Fushman ${ }^{12}$, Obioma Pelka ${ }^{15}$, \\ Christoph M. Friedrich ${ }^{15}$, Yashin Dicente Cid $^{16}$, Serge Kozlovski ${ }^{17}$, \\ Vitali Liauchuk ${ }^{17}$, Vassili Kovalev ${ }^{17}$, Raul Berari ${ }^{18}$, Paul Brie ${ }^{18}$, \\ Dimitri Fichou ${ }^{18}$, Mihai Dogariu ${ }^{1}$, Liviu Daniel Stefan ${ }^{1}$, \\ and Mihai Gabriel Constantin ${ }^{1}$ \\ 1 University Politehnica of Bucharest, Bucharest, Romania \\ bogdan.ionescu@upb.ro \\ 2 University of Applied Sciences Western Switzerland (HES-SO), \\ Delémont, Switzerland \\ 3 University of La Rochelle, La Rochelle, France \\ ${ }^{4}$ University of Bergen, Bergen, Norway \\ 5 Dublin City University, Dublin, Ireland \\ ${ }^{6}$ Pluribus One \& University of Cagliari, Cagliari, Italy \\ 7 SimulaMet, Oslo, Norway \\ 8 University of Science, Ho Chi Minh City, Vietnam \\ 9 Klagenfurt University, Klagenfurt, Austria \\ 10 University of Essex, Colchester, UK \\ 11 Wellcome Trust, London, UK \\ 12 National Library of Medicine, Bethesda, USA \\ 13 Philips Research Cambridge, Cambridge, USA \\ ${ }^{14}$ CVS Health, Monroeville, USA \\ 15 University of Applied Sciences and Arts Dortmund, Dortmund, Germany \\ 16 University of Warwick, Coventry, UK \\ 17 United Institute of Informatics Problems, Minsk, Belarus \\ 18 teleportHQ, Cluj-Napoca, Romania
}

\begin{abstract}
This paper presents an overview of the 2020 ImageCLEF lab that will be organized as part of the Conference and Labs of the Evaluation Forum - CLEF Labs 2020 in Thessaloniki, Greece. ImageCLEF is an ongoing evaluation initiative (run since 2003) that promotes the evaluation of technologies for annotation, indexing and retrieval of visual data with the aim of providing information access to large collections of images in various usage scenarios and domains. In 2020, the 18th edition of ImageCLEF will organize four main tasks: (i) a Lifelog task (videos, images and other sources) about daily activity understanding, retrieval
\end{abstract}


and summarization, (ii) a Medical task that groups three previous tasks (caption analysis, tuberculosis prediction, and medical visual question answering) with new data and adapted tasks, (iii) a Coral task about segmenting and labeling collections of coral images for 3D modeling, and a new (iv) Web user interface task addressing the problems of detecting and recognizing hand drawn website UIs (User Interfaces) for generating automatic code. The strong participation, with over 235 research groups registering and 63 submitting over 359 runs for the tasks in 2019 shows an important interest in this benchmarking campaign. We expect the new tasks to attract at least as many researchers for 2020 .

Keywords: Lifelogging retrieval and summarization - Medical image classification - Coral image segmentation and classification • Recognition of hand drawn website UIs - ImageCLEF benchmarking • Annotated data

\section{Introduction}

The ImageCLEF evaluation campaign was started as part of the CLEF (Cross Language Evaluation Forum) in 2003 [7,8]. It has been held every year since then and delivered many results in the analysis and retrieval of images [20,21]. Medical tasks started in 2004 and have in some years been the majority of the tasks in ImageCLEF $[18,19]$.

The objectives of ImageCLEF have always been the multilingual or languageindependent analysis of visual content. A focus has often been on multimodal data sets, so combining images with structured information, free text or other information that helps in the decision making.

Since 2018, ImageCLEF uses the crowdAI (now migrated to AIcrowd ${ }^{1}$ starting with 2020) platform to distribute the data and receive the submitted results. The system allows having an online leader board and gives the possibility to keep data sets accessible beyond competition, including a continuous submission of runs and addition to the leader board.

Over the years, ImageCLEF and also CLEF have shown a strong scholarly impact that was captured in $[27,28]$. This underlines the importance of evaluation campaigns for disseminating best scientific practices.

In the following, we introduce the four tasks that are going to run in the 2020 edition $^{2}$, namely: ImageCLEFlifelog, ImageCLEFmedical, ImageCLEFcoral, and the new ImageCLEFdrawnUI. Figure 1 captures with a few images the specificity of the tasks.

\footnotetext{
${ }^{1}$ https://www.aicrowd.com/.

${ }^{2}$ https://www.imageclef.org/2020.
} 

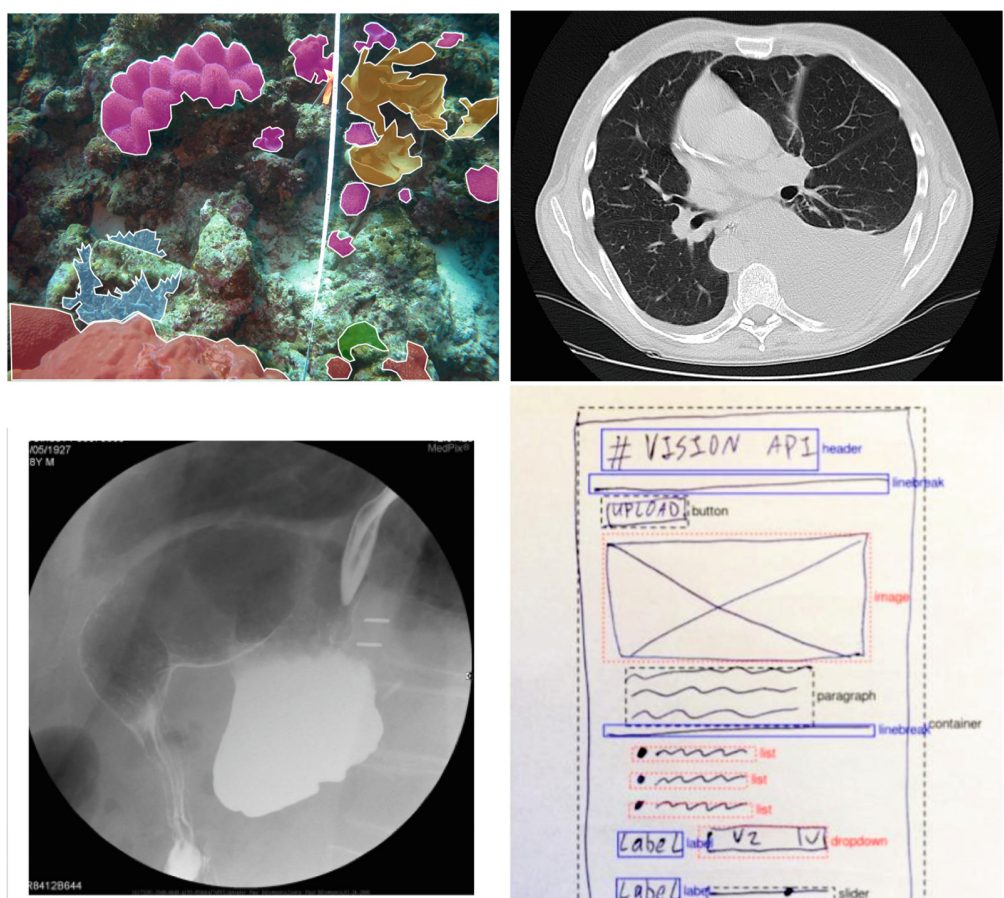

(h) Q: what is abnormal in the gastrointestinal image? A: gastric volvulus (organoaxial)

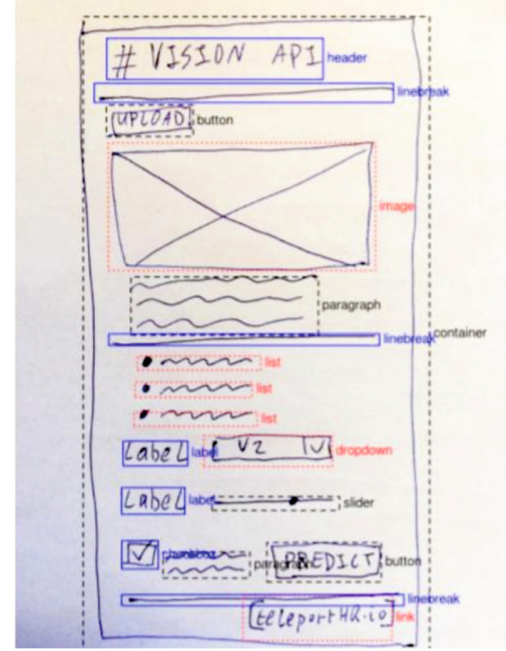

Fig. 1. Sample images from (left to right, top to bottom): ImageCLEFcoral with coral image segmentation and labeling, ImageCLEFmedical, e.g., tuberculosis prediction and Visual Question Answering, and ImageCLEFdrawnUI with recognition of hand drawn website UIs.

\section{ImageCLEFlifelog}

The main goal of the Lifelog task since its first edition [9] has been to advance the state-of-the-art research in lifelogging as an application of information retrieval. Different personal devices, such as smartphones, video cameras as well as wearable devices that allow collection of different data about our daily life are available. Large amounts of data are created by these devices containing videos, images, audio and sensor data. To be able to organize such a vast amount of data, there is a clear need of systems that can do this automatically.

As in the previous three editions, the task focuses mainly on images. The 2020 task will again be split into two subtasks: the lifelog moment retrieval and a new sports performance lifelog task. The first subtask includes new and enriched data, focusing on daily living activities and the chronological order of the moments. The second subtask provides a completely new dataset for assessing sports performance. 
For the Lifelog Core Task: Lifelog Moment Retrieval the participants are required to retrieve several predefined activities in a lifelogger's life. For example, they are asked to return the relevant moments for the query "Find the moment(s) when the lifelogger was having a beer on the beach with his/her friends". Particular attention will be paid to the diversification of the selected moments with respect to the target scenario. To make the task possible and interesting a rich multimodal dataset will be used. The data are completely new and contain about 4.5 months of data from three lifeloggers, 1,500-2,500 images per day, visual concepts, semantic content, biometric information, music listening history and computer usage.

The other task, Lifelog Task: Sports Performance Lifelog (SPLL, 1st edition), is completely new in terms of data and topic. Teams are required to predict the expected performance (e.g., estimated finishing time, average heart rate and other performance measurements) for a non-professional athlete who trained for a sports event. For the task, a new dataset is provided containing information collected from 20-24 people that train for a $5 \mathrm{~km}$ run. Objective sensor data is collected using the FitBit Versa 2 sport watch ${ }^{3}$; subjective wellness, training load and injury data is collected using the PMSYS system ${ }^{4}$; and information about meals, drinks, medication, etc. is collected using Google Forms. The data contain information about daily sleeping patterns, daily heart rate, sport activities, logs of food consumed during the training period (from at least 2 participants) and self reported data like mode, stress, fatigue, readiness to train and other measurements also used for professional soccer teams. The data are collected over a period of four to five month. The copyright and ethical approval to release the data are obtained by the task organizers. For the sports task data, we have the data approved by the Norwegian Center for Research Data ${ }^{5}$. For assessing the performance of the approaches, classic metrics will be used, e.g., precision, cluster recall (to account for the diversification), etc. For this sports task, we will also utilize metrics such as Mean Absolute Error (MAE) and Root mean squared error (RMSE).

\section{$3 \quad$ ImageCLEFmedical}

The ImageCLEF medical task has been running every year since 2004 [22]. In 2020, it will follow a similar format as in the previous edition [18] containing the same three subtasks with some modifications. The three tasks will be: tuberculosis analysis [10-12], figure caption analysis [13,17,23], and Visual Question Answering $[2,16]$.

The tuberculosis task will use, as in previous editions, Computed Tomography (CT) scans of patients with tuberculosis and more clinical data. In this edition, the task will concentrate only on generating an automatic report based on the CT and not assessing a TB severity score. The new report will be more detailed

\footnotetext{
3 https://www.fitbit.com/versa.

${ }^{4}$ https://forzasys.com/pmsys.html.

${ }^{5}$ https://nsd.no.
} 
than in the previous edition, containing more specific information, such as in which region each TB-related finding is located.

The caption analysis task will include more data compared to 2019. In 2020, an extension of the Radiology Objects in Context (ROCO) [24] data set is used and manually curated to reduce the data variability. The collection includes images from the medical literature including caption information, concepts and 7 sub-classes denoting the image radiology modality. The task concentrates on extracting Unified Medical Language System (UMLSß) Concept Unique Identifiers (CUIs) and can also be used as a first step towards the Medical Visual Question Answering (VQA-Med) task.

The medical Visual Question Answering (VQA-Med) task poses a challenging problem that involves both natural language processing and computer vision. In continuation of the two previous editions, the task consists of answering a natural language question from the visual content of an associated radiology image. VQA-Med 2020 will focus further on questions about abnormalities and will include a new subtask on visual question generation from radiology images.

\section{ImageCLEFcoral}

Coral reefs are important ecosystems because they are the most biodiverse parts of the oceans. However, corals thrive in narrow temperature ranges and ocean warming trends, among other factors, indicate that many of them will be lost within the next 30 years [3]. This would be a catastrophe, not only because of the extinction of many of the marine species they host but also because they provide an income and an essential food source to the people who live nearby $[4,25]$. Monitoring changes in reef composition and structural complexity on a large-scale is crucial to understanding and prioritizing conservation efforts.

Key to conservation work is knowledge of the state of reefs. Autonomous underwater vehicles are able to collect large amounts of data, more than can be annotated by a human. Although there have been promising attempts at automatically annotating imagery of reefs for complexity and benthic composition $[15,26]$, it is fair to say that the problem is far from being solved. The aim of this competition is to encourage researchers to improve techniques for automatically identifying areas of interest and label them in a way that helps marine biologists and ecologists.

Following the success of the first edition of the ImageCLEFcoral task [5], in 2020, participants are required to devise and implement algorithms for automatically annotating regions in a collection of images with types of benthic substrate, such as hard coral or sponge. The dataset comprises 440 humanannotated training images and a further 200 unseen test images of a region of coral reef in Indonesia. The images were captured in high-quality JPEG format using an innovative underwater image capture system developed at the Marine Technology Research Unit at the University of Essex, UK.

The ground truth annotations of the training and test sets were made largely by marine biology MSc students at Essex and checked by an experienced coral 
reef researcher. The annotations were performed using a web-based tool developed in a collaborative project with London-based company Filament Ltd which allowed many people to work concurrently and which was carefully designed to be simple and quick to use; this proved so effective that we are exploring whether the tool can be made publicly available for other tasks in the future.

As in the first edition, algorithmic performance will be evaluated on the unseen test data using the intersection over union metric popularized in the PASCAL VOC 6 exercise. This computes the area of intersection of the output of an algorithm and the corresponding ground truth, normalizing that by the area of their union to ensure its maximum value remains bounded.

\section{ImageCLEFdrawnUI}

The user interface (UI) is the space where interactions between humans and computers occur. The increasing dependence of web and mobile applications have led many enterprises to increase the priority of developing user interfaces, in an effort to improve the overall user experience. Currently, the performance of any modern digital product is strongly correlated to the quality and usability of its user interface. However, building a user interface for digital applications is a complex process involving the interaction between multiple specialists, each with its own specific domain knowledge.

Generally, a business owner is setting a business goal. Then, a project owner or product owner is refining the requirements and builds a prototype of the application using wireframes. Once validated, the designer transforms the wireframes into designs which are transformed into code by the developer. This process is time consuming and expensive. Moreover, as more people get involved, the process is increasingly error prone. In addition, user interface experts are in limited supply. Globally, there are about 22 million developers ${ }^{7}$, among which only 10 million are estimated to be also JavaScript UI developers ${ }^{8}$.

Recently the use of machine learning to facilitate this process has been demonstrated as a viable solution. In 2018, pix2code, a machine learning based approach to generate low fidelity domain specific languages from screenshots, was published and open sourced [1]. Also, in 2018 Chen Chunyang et al. created their own dataset from android apps with 185,277 pairs of UI images and GUI skeletons. The dataset and code were also open-sourced [6].

In this context, in the 2020 ImageCLEFdrawnUI task, given a set of images of hand drawn UIs, participants are required to develop machine learning techniques that are able to predict the exact position and type of UI elements. The provided dataset consists of 3,000 hand drawn images inspired from mobile application screenshots and actual web pages containing 1,000 different templates. Each image was manually labelled with the positions of the bounding

\footnotetext{
${ }^{6}$ http://host.robots.ox.ac.uk/pascal/VOC/.

${ }^{7}$ http://evansdata.com.

${ }^{8}$ http://appdevelopermagazine.com.
} 
boxes corresponding to each UI element and its type. To avoid any ambiguity, a predefined shape dictionary with 21 classes is used (e.g., paragraph, label, header). The performance of the algorithms will be evaluated using the standard mean Average Precision over IoU .5, commonly used in object detection [14].

\section{Conclusions}

In this paper, we present an overview of the upcoming ImageCLEF 2020 campaign. ImageCLEF has organized many tasks in a variety of domains over the past 18 years, from general stock photography, medical and biodiversity data to multimodal lifelogging. The focus has always been on language independent or multi-lingual approaches and most often on multimodal data analysis. 2020 has a set of interesting tasks that are expected to again draw a large number of participants. As in 2019, the focus for 2020 has been on the diversity of applications and on creating clean data sets to provide a solid basis for the evaluations.

Acknowledgement. Mihai Dogariu and Liviu Daniel Stefan work has been funded by the Operational Programme Human Capital of the Ministry of European Funds through the Financial Agreement 51675/09.07.2019, SMIS code 125125.

\section{References}

1. Beltramelli, T.: pix2code: generating code from a graphical user interface screenshot. In: Proceedings of the ACM SIGCHI Symposium on Engineering Interactive Computing Systems, pp. 1-9 (2018)

2. Ben Abacha, A., Hasan, S.A., Datla, V.V., Liu, J., Demner-Fushman, D., Müller, H.: VQA-Med: overview of the medical visual question answering task at ImageCLEF 2019. In: Working Notes of CLEF 2019 - Conference and Labs of the Evaluation Forum, Lugano, Switzerland, 9-12 September 2019 (2019). http://ceur-ws. org/Vol-2380/paper_272.pdf

3. Birkeland, C.: Global status of coral reefs: in combination, disturbances and stressors become ratchets. In: World Seas: An Environmental Evaluation, pp. 35-56. Elsevier, Amsterdam (2019)

4. Brander, L.M., Rehdanz, K., Tol, R.S., Van Beukering, P.J.: The economic impact of ocean acidification on coral reefs. Clim. Chang. Econ. 3(01), 1250002 (2012)

5. Chamberlain, J., Campello, A., Wright, J.P., Clift, L.G., Clark, A., García Seco de Herrera, A.: Overview of ImageCLEFcoral 2019 task. In: CLEF2019 Working Notes. CEUR Workshop Proceedings, vol. 2380. CEUR-WS.org (2019)

6. Chen, C., Su, T., Meng, G., Xing, Z., Liu, Y.: From UI design image to GUISkeleton: a neural machine translator to bootstrap mobile GUI implementation. In: International Conference on Software Engineering, vol. 6 (2018)

7. Clough, P., Müller, H., Sanderson, M.: The CLEF 2004 cross-language image retrieval track. In: Peters, C., Clough, P., Gonzalo, J., Jones, G.J.F., Kluck, M., Magnini, B. (eds.) CLEF 2004. LNCS, vol. 3491, pp. 597-613. Springer, Heidelberg (2005). https://doi.org/10.1007/11519645_59

8. Clough, P., Sanderson, M.: The CLEF 2003 cross language image retrieval task. In: Proceedings of the Cross Language Evaluation Forum (CLEF 2003) (2004) 
9. Dang-Nguyen, D.T., Piras, L., Riegler, M., Boato, G., Zhou, L., Gurrin, C.: Overview of ImageCLEFlifelog 2017: lifelog retrieval and summarization. In: Cappellato, L., Ferro, N., Goeuriot, L., Mandl, T. (eds.) CLEF 2017 Working Notes. CEUR Workshop Proceedings (CEUR-WS.org) http://ceur-ws.org/Vol1866/ (2017). ISSN 1613-0073

10. Dicente Cid, Y., Kalinovsky, A., Liauchuk, V., Kovalev, V., Müller, H.: Overview of ImageCLEFtuberculosis 2017 - predicting tuberculosis type and drug resistances. In: CLEF2017 Working Notes. CEUR Workshop Proceedings, Dublin, Ireland, 1114 September 2017. CEUR-WS.org http://ceur-ws.org

11. Dicente Cid, Y., Liauchuk, V., Klimuk, D., Tarasau, A., Kovalev, V., Müller, H.: Overview of ImageCLEF tuberculosis 2019 - automatic CT-based report generation and tuberculosis severity assessment. In: CLEF2019 Working Notes. CEUR Workshop Proceedings, Lugano, Switzerland, 9-12 September 2019. CEUR-WS.org http://ceur-ws.org

12. Dicente Cid, Y., Liauchuk, V., Kovalev, V., Müller, H.: Overview of ImageCLEFtuberculosis 2018 - detecting multi-drug resistance, classifying tuberculosis type, and assessing severity score. In: CLEF2018 Working Notes. CEUR Workshop Proceedings, Avignon, France, 10-14 September 2018. CEUR-WS.org http://ceur-ws. org

13. Eickhoff, C., Schwall, I., García Seco de Herrera, A., Müller, H.: Overview of ImageCLEFcaption 2017 - the image caption prediction and concept extraction tasks to understand biomedical images. In: CLEF2017 Working Notes. CEUR Workshop Proceedings, Dublin, Ireland, 11-14 September 2017. CEUR-WS.org http://ceurws.org

14. Everingham, M., Gool, L.V., Williams, C.K.I., Winn, J., Zisserman, A.: The PASCAL Visual Object Classes (VOC ) challenge. Int. J. Comput. Vis. 88, 303-338 (2010). https://doi.org/10.1007/s11263-009-0275-4

15. Gonzalez-Rivero, M., et al.: The Catlin Seaview Survey-kilometre-scale seascape assessment, and monitoring of coral reef ecosystems. Aquat. Conserv. Mar. Freshwater Ecosyst. 24, 184-198 (2014)

16. Hasan, S.A., Ling, Y., Farri, O., Liu, J., Lungren, M., Müller, H.: Overview of the ImageCLEF 2018 medical domain visual question answering task. In: CLEF 2018 Working Notes. CEUR Workshop Proceedings, Avignon, France, 10-14 September 2018. CEUR-WS.org http://ceur-ws.org

17. Seco de Herrera, A.G., Eickhoff, C., Andrearczyk, V., Müller, H.: Overview of the ImageCLEF 2018 caption prediction tasks. In: CLEF 2018 Working Notes. CEUR Workshop Proceedings, Avignon, France, 10-14 September 2018. CEUR-WS.org http://ceur-ws.org

18. Ionescu, B., et al.: ImageCLEF 2019: multimedia retrieval in medicine, lifelogging, security and nature. In: Crestani, F., et al. (eds.) CLEF 2019. LNCS, vol. 11696, pp. 358-386. Springer, Cham (2019). https://doi.org/10.1007/978-3-030-28577-7_28

19. Ionescu, B., et al.: Overview of ImageCLEF 2018: challenges, datasets and evaluation. In: Bellot, P., et al. (eds.) CLEF 2018. LNCS, vol. 11018, pp. 309-334. Springer, Cham (2018). https://doi.org/10.1007/978-3-319-98932-7_28

20. Kalpathy-Cramer, J., de Herrera, A.G.S., Demner-Fushman, D., Antani, S., Bedrick, S., Müller, H.: Evaluating performance of biomedical image retrieval systems: overview of the medical image retrieval task at ImageCLEF 2004-2014. Comput. Med. Imaging Graph. 39(0), 55-61 (2015)

21. Müller, H., Clough, P., Deselaers, T., Caputo, B. (eds.): ImageCLEF - Experimental Evaluation in Visual Information Retrieval. INRED, vol. 32. Springer, Heidelberg (2010). https://doi.org/10.1007/978-3-642-15181-1 
22. Müller, H., Kalpathy-Cramer, J., García Seco de Herrera, A.: Experiences from the ImageCLEF medical retrieval and annotation tasks. Information Retrieval Evaluation in a Changing World. TIRS, vol. 41, pp. 231-250. Springer, Cham (2019). https://doi.org/10.1007/978-3-030-22948-1_10

23. Pelka, O., Friedrich, C.M., García Seco de Herrera, A., Müller, H.: Overview of the ImageCLEFmed 2019 concept prediction task. In: CLEF2019 Working Notes. CEUR Workshop Proceedings, Lugano, Switzerland, 09-12 September 2019, vol. 2380. CEUR-WS.org http://ceur-ws.org

24. Pelka, O., Koitka, S., Rückert, J., Nensa, F., Friedrich, C.M.: Radiology objects in context (ROCO): a multimodal image dataset. In: Stoyanov, D., et al. (eds.) LABELS/CVII/STENT -2018. LNCS, vol. 11043, pp. 180-189. Springer, Cham (2018). https://doi.org/10.1007/978-3-030-01364-6_20

25. Speers, A.E., Besedin, E.Y., Palardy, J.E., Moore, C.: Impacts of climate change and ocean acidification on coral reef fisheries: an integrated ecological-economic model. Ecol. Econ. 128, 33-43 (2016)

26. Stokes, M., Deane, G.: Automated processing of coral reef benthic images. Limnol. Oceanogr. Methods 7, 157-168 (2009)

27. Tsikrika, T., de Herrera, A.G.S., Müller, H.: Assessing the scholarly impact of ImageCLEF. In: Forner, P., Gonzalo, J., Kekäläinen, J., Lalmas, M., de Rijke, M. (eds.) CLEF 2011. LNCS, vol. 6941, pp. 95-106. Springer, Heidelberg (2011). https://doi.org/10.1007/978-3-642-23708-9_12

28. Tsikrika, T., Larsen, B., Müller, H., Endrullis, S., Rahm, E.: The scholarly impact of CLEF (2000-2009). In: Forner, P., Müller, H., Paredes, R., Rosso, P., Stein, B. (eds.) CLEF 2013. LNCS, vol. 8138, pp. 1-12. Springer, Heidelberg (2013). https:// doi.org/10.1007/978-3-642-40802-1_1 